QUARTERLY OF APPLIED MATHEMATICS

VOLUME LXIII, NUMBER 4

DECEMBER 2005, PAGES 601-618

S $0033-569 X(05) 00971-2$

Article electronically published on July 26, 2005

\title{
THE DIRECT MEG PROBLEM IN THE PRESENCE OF AN ELLIPSOIDAL SHELL INHOMOGENEITY
}

\author{
BY \\ GEORGE DASSIOS (Division of Applied Mathematics, Department of Chemical Engineering, \\ University of Patras, and ICEHT/FORTH) \\ AND \\ FOTINI KARIOTOU (Division of Applied Mathematics, Department of Chemical Engineering, \\ University of Patras, and Hellenic Open University)
}

\begin{abstract}
The forward problem of Magnetoencephalography for an ellipsoidal inhomogeneous shell-model of the brain is considered. The inhomogeneity enters through a confocal ellipsoidal shell exhibiting different conductivity than the one of the brain tissue. It is shown that, as far as the leading quadrupolic moment of the exterior magnetic field is concerned, the complicated expression associated with the field itself is the same as in the homogeneous case, while the effect of the shell is focused on the form of the generalized dipole moment. In contrast to the spherical case, where no shell inhomogeneities are "readable" outside the skull, the ellipsoidal shells establish their existence on the exterior magnetic induction field in a way that depends not only on the geometry but also on the conductivity of the shell. The degenerated spherical results are fully recovered.
\end{abstract}

1. Introduction. The mathematical theory of Electroencephalography (EEG) and Magnetoencephalography (MEG) was founded in the late 60s, mainly on the basis of the works of Geselowitz [7, 8. Since then, many efforts have been made to produce analytic solutions for the related direct problem where the field generated by a given source is sought. We mention the works of Ilmoniemi, Hämäläinen and Knuutila [10, Sarvas [15] and Fokas, Kurylev and Marinakis [5] for the spherical brain model, the works of Cuffin and Cohen [1] and de Munck [6] for the spheroidal brain model and the work of Nolte, Fieseler and Curio [14] for perturbative models of the brain. But, as anatomy indicates, the actual geometry of the human brain is best approximated by a triaxial ellipsoid [16], a geometrical shape far more complicated than the sphere or even the spheroid. Intense efforts towards a complete analytic solution for EEG and MEG problems in ellipsoidal geometry led to results included in [3, 4, 11, 12. The present work aims in obtaining an analytic expression of the leading quadrupolic term for the exterior magnetic field in the

Received August 13, 2004.

2000 Mathematics Subject Classification. Primary 78M99, 35QXX.

(C)2005 Brown University 
presence of an ellipsoidal shell with different conductivity from the one characterizing the brain tissue.

The result is amazingly similar to the corresponding result of the single ellipsoidal model [3. The only effect that the inhomogeneous shell has on the form of the magnetic field, is attributed to the expression of the generalized dipole moment appearing in the quadrupole term. As we show, this generalized dipole moment is a complicated expression of the conductivity profiles in the two compartments as well as of the semiaxes of the brain and its covering shell.

Everything connected to the geometry of the problem is expressed in terms of canonical dyadics. It is important to realize that the form of the generalized dipole moment dictates the orientation of the silent sources which are drastically effected by the conductive ellipsoidal shell. It is also shown that the part of the solution associated with the observation point, where the magnetic field is measured, is not influenced by the existence of the shell.

Section 2 involves the mathematical statement of the direct MEG problem for an ellipsoidal brain which is excited by a current dipole in its interior and it is surrounded by a confocal ellipsoidal shell of different conductivity. In order to solve this problem we need to solve first the related EEG problem, a task that has been fulfilled in 11]. The main result of the present work is exposed in Section 3 where the exterior magnetic field is evaluated through a series of appropriate manipulations with elliptic integrals. In Section 4 we reduce the ellipsoidal results to the corresponding spherical ones as well as to the case of the single component homogeneous brain model.

2. Statement of the problem. First we define the geometry of the problem. The brain is modeled with the triaxial ellipsoid

$$
\frac{x_{1}^{2}}{b_{1}^{2}}+\frac{x_{2}^{2}}{b_{2}^{2}}+\frac{x_{3}^{2}}{b_{3}^{2}} \leq 1
$$

which has the constant conductivity $\sigma_{b}$. We denote this region by $V_{b}$ and let $S_{b}=\partial V_{b}$. The confocal ellipsoidal shell $V_{\alpha}$, bounded by the ellipsoid $S_{b}$ and the ellipsoid $S_{\alpha}$ given by

$$
\frac{x_{1}^{2}}{\alpha_{1}^{2}}+\frac{x_{2}^{2}}{\alpha_{2}^{2}}+\frac{x_{3}^{2}}{\alpha_{3}^{2}}=1
$$

surrounds the brain and is characterized by the conductivity $\sigma_{\alpha} \neq \sigma_{b}$. The space $\mathrm{V}_{\alpha}$ models either the cerebrospinal fluid, the bone, the skin, or an average of all of them. The semiaxes are ordered as follows:

$$
\left.\begin{array}{l}
\alpha_{3}<\alpha_{2}<\alpha_{1} \\
b_{3}<b_{2}<b_{1}<\alpha_{1}
\end{array}\right\}
$$


In terms of the ellipsoidal coordinates $(\rho, \mu, \nu)[9$, which are connected to the Cartesian coordinates $\left(x_{1}, x_{2}, x_{3}\right)$ by

$$
\left.\begin{array}{l}
h_{2} h_{3} x_{1}=\rho \mu \nu, \\
h_{1} h_{3} x_{2}=\sqrt{\rho^{2}-h_{3}^{2}} \sqrt{\mu^{2}-h_{3}^{2}} \sqrt{h_{3}^{2}-\nu^{2}}, \\
h_{1} h_{2} x_{3}=\sqrt{\rho^{2}-h_{2}^{2}} \sqrt{h_{2}^{2}-\mu^{2}} \sqrt{h_{2}^{2}-\nu^{2}},
\end{array}\right\}
$$

where the semifocal distances $h_{1}, h_{2}, h_{3}$ are given by

$$
\left.\begin{array}{l}
h_{1}^{2}=\alpha_{2}^{2}-\alpha_{3}^{2}=b_{2}^{2}-b_{3}^{2}, \\
h_{2}^{2}=\alpha_{1}^{2}-\alpha_{3}^{2}=b_{1}^{2}-b_{3}^{2}, \\
h_{3}^{2}=\alpha_{1}^{2}-\alpha_{2}^{2}=b_{1}^{2}-b_{2}^{2},
\end{array}\right\}
$$

the surface $\mathrm{S}_{b}$ corresponds to $\rho=b_{1}$, the surface $\mathrm{S}_{\alpha}$ to $\rho=\alpha_{1}$, the core-domain $\mathrm{V}_{b}$ to $\rho \in\left[h_{2}, b_{1}\right)$, and the shell-domain $\mathrm{V}_{\alpha}$ to $\rho \in\left(b_{1}, \alpha_{1}\right)$. The exterior domain $\mathrm{V}$ is then described by $\rho>\alpha_{1}$ and has zero conductivity. The magnetic permeability $\mu_{0}$ is taken to be constant in all of $\mathbb{R}^{3}$.

At the point $\mathbf{r}_{0}=\left(\rho_{0}, \mu_{0}, \nu_{0}\right)$, being within $\mathrm{V}_{b}$, an equivalent dipole current source with dipole moment $\mathbf{Q}$ models the local electrochemical activity of the neurons.

Within the framework of quasistatic electromagnetic theory [13, which is generally accepted for MEG problems [15, this current

$$
\mathbf{J}(\mathbf{r})=\mathbf{Q} \delta\left(\mathbf{r}-\mathbf{r}_{0}\right)
$$

gives rise to an electric potential $\mathrm{u}$ and to a magnetic induction field $\mathbf{B}$.

Let us denote by $\mathrm{u}_{b}, \mathrm{u}_{\alpha}$ and $\mathrm{u}$ the electric field in $\mathrm{V}_{b}, \mathrm{~V}_{\alpha}$ and $\mathrm{V}$, respectively. Then the magnetic field $\mathbf{B}$, generated in $\mathrm{V}$, is a consequence of the primary current $\mathbf{J}$ and the induction currents $-\sigma_{\alpha} \nabla u_{\alpha}$ and $-\sigma_{b} \nabla u_{b}$ which are supported in $\mathrm{V}_{\alpha}$ and $\mathrm{V}_{b}$, respectively.

Hence, Ampere's law [13.

$$
\mathbf{B}(\mathbf{r})=\frac{\mu_{0}}{4 \pi} \int_{G} \mathbf{J}^{t}\left(\mathbf{r}^{\prime}\right) \times \frac{\mathbf{r}-\mathbf{r}^{\prime}}{\left|\mathbf{r}-\mathbf{r}^{\prime}\right|^{3}} d v\left(\mathbf{r}^{\prime}\right)
$$

where $G$ stands for the support of the total current $\mathbf{J}^{t}$, implies the representation

$$
\begin{aligned}
\mathbf{B}(\mathbf{r})= & \frac{\mu_{0}}{4 \pi} \mathbf{Q} \times \frac{\mathbf{r}-\mathbf{r}_{0}}{\left|\mathbf{r}-\mathbf{r}_{0}\right|^{3}}-\frac{\mu_{0} \sigma_{\alpha}}{4 \pi} \int_{V_{\alpha}}\left(\nabla_{\mathbf{r}^{\prime}} u_{\alpha}\left(\mathbf{r}^{\prime}\right)\right) \times\left(\nabla_{\mathbf{r}^{\prime}} \frac{1}{\left|\mathbf{r}-\mathbf{r}^{\prime}\right|}\right) d v\left(\mathbf{r}^{\prime}\right) \\
& -\frac{\mu_{0} \sigma_{b}}{4 \pi} \int_{V_{b}}\left(\nabla_{\mathbf{r}^{\prime}} u_{b}\left(\mathbf{r}^{\prime}\right)\right) \times\left(\nabla_{\mathbf{r}^{\prime}} \frac{1}{\left|\mathbf{r}-\mathbf{r}^{\prime}\right|}\right) d v\left(\mathbf{r}^{\prime}\right)
\end{aligned}
$$

or, in view of Geselowitz formula [2],

$$
\begin{aligned}
\mathbf{B}(\mathbf{r})= & \frac{\mu_{0}}{4 \pi} \mathbf{Q} \times \frac{\mathbf{r}-\mathbf{r}_{0}}{\left|\mathbf{r}-\mathbf{r}_{0}\right|^{3}}-\frac{\mu_{0} \sigma_{\alpha}}{4 \pi} \int_{S_{\alpha}} u_{\alpha}\left(\mathbf{r}^{\prime}\right) \hat{\boldsymbol{\rho}}^{\prime} \times\left(\nabla_{\mathbf{r}^{\prime}} \frac{1}{\left|\mathbf{r}-\mathbf{r}^{\prime}\right|}\right) d s\left(\mathbf{r}^{\prime}\right) \\
& +\frac{\mu_{0}}{4 \pi} \int_{S_{b}}\left(\sigma_{\alpha} u_{\alpha}\left(\mathbf{r}^{\prime}\right)-\sigma_{b} u_{b}\left(\mathbf{r}^{\prime}\right)\right) \hat{\boldsymbol{\rho}}^{\prime} \times\left(\nabla_{\mathbf{r}^{\prime}} \frac{1}{\left|\mathbf{r}-\mathbf{r}^{\prime}\right|}\right) d s\left(\mathbf{r}^{\prime}\right)
\end{aligned}
$$


where $\hat{\boldsymbol{\rho}}^{\prime}$ denotes the outward unit normal on the corresponding ellipsoid.

Representation (2.9) recasts the contribution of the conductive domains to $\mathbf{B}$ in the form of surface distributions of dipoles oriented normally to the interfaces $\mathrm{S}_{\alpha}$ and $\mathrm{S}_{b}$. The density of these dipole distributions is proportional to $-\sigma_{\alpha} u_{\alpha}$ on $\mathrm{S}_{\alpha}$ and to $\sigma_{\alpha} u_{\alpha}-\sigma_{b} u_{b}$ on $\mathrm{S}_{b}$. Therefore, our first task is to solve the following boundary value problem, for the electric potential

$$
\begin{aligned}
\Delta u(\mathbf{r}) & =0, \quad \mathbf{r} \in V, \\
\Delta u_{\alpha}(\mathbf{r}) & =0, \quad \mathbf{r} \in V_{\alpha}, \\
\Delta u_{b}(\mathbf{r}) & =\frac{1}{\sigma_{b}} \nabla \cdot \mathbf{J}(\mathbf{r}), \quad \mathbf{r} \in V_{b},
\end{aligned}
$$

where $\mathbf{J}(\mathbf{r})$ is given by (2.6).

Continuity conditions demand that the fields $\mathrm{u}, \mathrm{u}_{\alpha}, \mathrm{u}_{b}$ are connected through the surface conditions

$$
\begin{aligned}
u_{\alpha}(\mathbf{r}) & =u(\mathbf{r}), \quad \mathbf{r} \in S_{\alpha}, \\
\partial_{n} u_{\alpha}(\mathbf{r}) & =0, \quad \mathbf{r} \in S_{\alpha}, \\
u_{\alpha}(\mathbf{r}) & =u_{b}(\mathbf{r}), \quad \mathbf{r} \in S_{b}, \\
\sigma_{\alpha} \partial_{n} u_{\alpha}(\mathbf{r}) & =\sigma_{b} \partial_{n} u_{b}(\mathbf{r}), \quad \mathbf{r} \in S_{b},
\end{aligned}
$$

where $\partial_{n}$ stands for the outward normal differentiation on the corresponding surface.

Furthermore, the exterior electric field has to satisfy the asymptotic condition

$$
u(\mathbf{r})=O\left(\frac{1}{r}\right), \quad r \rightarrow \infty .
$$

The solution of the problem (2.10)-(2.17), which has been obtained in [11], assumes the following appropriate-for-our-purpose form:

$$
\begin{aligned}
u(\mathbf{r})= & d_{0}^{1} \frac{I_{0}^{1}(\rho)}{I_{0}^{1}\left(\alpha_{1}\right)}+\sum_{n=1}^{\infty} \sum_{m=1}^{2 n+1} \frac{\mathbf{Q} \cdot \nabla \mathbb{E}_{n}^{m}\left(\mathbf{r}_{0}\right)}{\gamma_{n}^{m} C_{n}^{m}} \\
& \times \frac{1}{E_{n}^{m}\left(\alpha_{1}\right) E_{n}^{m^{\prime}}\left(\alpha_{1}\right) \alpha_{2} \alpha_{3}} \frac{I_{n}^{m}(\rho)}{I_{n}^{m}\left(\alpha_{1}\right)} \mathbb{E}_{n}^{m}(\rho, \mu, \nu)
\end{aligned}
$$

for $\mathbf{r} \in V$,

$$
\begin{aligned}
u_{\alpha}(\mathbf{r})= & d_{0}^{1}+\sum_{n=1}^{\infty} \sum_{m=1}^{2 n+1} \frac{\mathbf{Q} \cdot \nabla \mathbb{E}_{n}^{m}\left(\mathbf{r}_{0}\right)}{\gamma_{n}^{m} C_{n}^{m}} \\
& \times\left(I_{n}^{m}(\rho)-I_{n}^{m}\left(\alpha_{1}\right)+\frac{1}{E_{n}^{m}\left(\alpha_{1}\right) E_{n}^{m^{\prime}}\left(\alpha_{1}\right) \alpha_{2} \alpha_{3}}\right) \mathbb{E}_{n}^{m}(\rho, \mu, \nu)
\end{aligned}
$$

for $\mathbf{r} \in V_{\alpha}$, and

$$
\begin{aligned}
u_{b}(\mathbf{r})= & d_{0}^{1}+\sum_{n=1}^{\infty} \sum_{m=1}^{2 n+1} \frac{\mathbf{Q} \cdot \nabla \mathbb{E}_{n}^{m}\left(\mathbf{r}_{0}\right)}{\gamma_{n}^{m} C_{n}^{m}}\left[\left(\frac{C_{n}^{m}}{\sigma_{b}}\left(I_{n}^{m}\left(b_{1}\right)-I_{n}^{m}(\rho)\right)\right.\right. \\
& \left.\left.+I_{n}^{m}\left(b_{1}\right)-I_{n}^{m}\left(\alpha_{1}\right)+\frac{1}{E_{n}^{m}\left(\alpha_{1}\right) E_{n}^{m^{\prime}}\left(\alpha_{1}\right) \alpha_{2} \alpha_{3}}\right)\right] \mathbb{E}_{n}^{m}(\rho, \mu, \nu)
\end{aligned}
$$


for $\mathbf{r} \in V_{b}$, where for each $n=1,2, \ldots$ and $m=1,2, \ldots, 2 n+1$,

$$
\begin{aligned}
C_{n}^{m}= & \sigma_{\alpha}+\left(\sigma_{b}-\sigma_{\alpha}\right) \\
& \times\left[\left(I_{n}^{m}\left(b_{1}\right)-I_{n}^{m}\left(\alpha_{1}\right)\right) E_{n}^{m}\left(b_{1}\right) E_{n}^{m^{\prime}}\left(b_{1}\right) b_{2} b_{3}+\frac{E_{n}^{m}\left(b_{1}\right) E_{n}^{m^{\prime}}\left(b_{1}\right) b_{2} b_{3}}{E_{n}^{m}\left(\alpha_{1}\right) E_{n}^{m^{\prime}}\left(\alpha_{1}\right) \alpha_{2} \alpha_{3}}\right]
\end{aligned}
$$

and $d_{0}^{1}$ is an arbitrary constant (as any potential function owes to have).

The rest of the variables appearing in (2.18)-(2.21) are connected to the ellipsoidal harmonics

$$
\begin{aligned}
& \mathbb{E}_{n}^{m}(\rho, \mu, \nu)=E_{n}^{m}(\rho) E_{n}^{m}(\mu) E_{n}^{m}(\nu), \\
\mathbb{F}_{n}^{m}(\rho, \mu, \nu)= & F_{n}^{m}(\rho) E_{n}^{m}(\mu) E_{n}^{m}(\nu)=(2 n+1) I_{n}^{m}(\rho) \mathbb{E}_{n}^{m}(\rho, \mu, \nu)
\end{aligned}
$$

given in terms of the Lamé functions $E_{n}^{m}$ and $F_{n}^{m}$ and the elliptic integrals $I_{n}^{m}(\rho)$ which are defined in the Appendix.

Finally, $\gamma_{n}^{m}$ denote the ellipsoidal normalization constants

$$
\gamma_{n}^{m}=\int_{\rho=\rho_{0}}\left[E_{n}^{m}(\mu) E_{n}^{m}(\nu)\right]^{2} l_{\rho_{0}}(\mu, \nu) d s(\mu, \nu)
$$

for each $n=1,2, \ldots$ and $m=1,2, \ldots, 2 n+1$, where $l_{\rho_{0}}(\mu, \nu)$ is the ellipsoidal weighting function defined by

$$
l_{\rho_{0}}(\mu, \nu)=\left[\left(\rho_{0}^{2}-\mu^{2}\right)\left(\rho_{0}^{2}-\nu^{2}\right)\right]^{-\frac{1}{2}} .
$$

3. The magnetic field. Our main task in this section is to find analytic expressions for the integrals appearing in (2.9). This will be achieved via appropriate transformations, the orthogonality of the surface ellipsoidal harmonics and the evaluation of the normalization integrals. We start with the evaluation of the integral

$$
\mathbf{I}_{\alpha}(\mathbf{r})=\int_{S_{\alpha}} u_{\alpha}\left(\mathbf{r}^{\prime}\right) \hat{\boldsymbol{\rho}}^{\prime} \times \nabla_{\mathbf{r}^{\prime}} \frac{1}{\left|\mathbf{r}-\mathbf{r}^{\prime}\right|} d s\left(\mathbf{r}^{\prime}\right) .
$$

Detailed analysis of the part $\hat{\boldsymbol{\rho}}^{\prime} \times \nabla_{\mathbf{r}^{\prime}}\left|\mathbf{r}-\mathbf{r}^{\prime}\right|^{-1}$ leads to the expansion 3 ]

$$
\begin{aligned}
\hat{\boldsymbol{\rho}}^{\prime} \times\left.\frac{\mathbf{r}-\mathbf{r}^{\prime}}{\left|\mathbf{r}-\mathbf{r}^{\prime}\right|^{3}}\right|_{\rho^{\prime}=\alpha_{1}}= & l_{\alpha}\left(\mu^{\prime}, \nu^{\prime}\right)\left[\sum_{m=1}^{3} \boldsymbol{\beta}_{m} E_{1}^{m}\left(\mu^{\prime}\right) E_{1}^{m}\left(\nu^{\prime}\right)\right. \\
& \left.+\sum_{m=1}^{5} \boldsymbol{\delta}_{m} E_{2}^{m}\left(\mu^{\prime}\right) E_{2}^{m}\left(\nu^{\prime}\right)\right]+\mathrm{O}\left(e l_{3}^{\prime}\right)
\end{aligned}
$$


where $l_{\alpha}$ is obtained from (2.25) by substituting $\rho_{0}=\alpha_{1}$,

$$
\begin{aligned}
& \boldsymbol{\beta}_{m}=3 \frac{\alpha_{1} \alpha_{2} \alpha_{3}}{h_{1} h_{2} h_{3}} \frac{h_{m}}{\alpha_{m}} \hat{\mathbf{x}}_{m} \otimes \mathbf{r} \times \tilde{\mathbf{H}}_{1}(\rho), \quad m=1,2,3, \\
& \boldsymbol{\delta}_{1}=-\frac{\alpha_{1} \alpha_{2} \alpha_{3}}{3\left(\Lambda_{\alpha}-\Lambda_{\alpha}^{\prime}\right)} \tilde{\mathbf{\Lambda}}_{\alpha} \times \tilde{\mathbf{F}}_{\alpha}(\mathbf{r}), \\
& \boldsymbol{\delta}_{2}=\frac{\alpha_{1} \alpha_{2} \alpha_{3}}{3\left(\Lambda_{\alpha}-\Lambda_{\alpha}^{\prime}\right)} \tilde{\mathbf{\Lambda}}_{\alpha}^{\prime} \times \tilde{\mathbf{F}}_{\alpha}(\mathbf{r}), \\
& \boldsymbol{\delta}_{3}=\frac{\alpha_{1} \alpha_{2} \alpha_{3}}{h_{1} h_{2} h_{3}^{2}}\left[\frac{\alpha_{2}}{\alpha_{1}} \hat{\mathbf{x}}_{1} \otimes \hat{\mathbf{x}}_{2}+\frac{\alpha_{1}}{\alpha_{2}} \hat{\mathbf{x}}_{2} \otimes \hat{\mathbf{x}}_{1}\right] \times \tilde{\mathbf{F}}_{\alpha}(\mathbf{r}), \\
& \boldsymbol{\delta}_{4}=\frac{\alpha_{1} \alpha_{2} \alpha_{3}}{h_{1} h_{2}^{2} h_{3}}\left[\frac{\alpha_{3}}{\alpha_{1}} \hat{\mathbf{x}}_{1} \otimes \hat{\mathbf{x}}_{3}+\frac{\alpha_{1}}{\alpha_{3}} \hat{\mathbf{x}}_{3} \otimes \hat{\mathbf{x}}_{1}\right] \times \tilde{\mathbf{F}}_{\alpha}(\mathbf{r}), \\
& \boldsymbol{\delta}_{5}=\frac{\alpha_{1} \alpha_{2} \alpha_{3}}{h_{1}^{2} h_{2} h_{3}}\left[\frac{\alpha_{3}}{\alpha_{2}} \hat{\mathbf{x}}_{2} \otimes \hat{\mathbf{x}}_{3}+\frac{\alpha_{2}}{\alpha_{3}} \hat{\mathbf{x}}_{3} \otimes \hat{\mathbf{x}}_{2}\right] \times \tilde{\mathbf{F}}_{\alpha}(\mathbf{r}),
\end{aligned}
$$

with the cross-dot product defined by

$$
(\mathbf{a} \otimes \mathbf{b}) \times(\mathbf{c} \otimes \mathbf{d})=(\mathbf{a} \times \mathbf{c})(\mathbf{b} \cdot \mathbf{d}),
$$

and the related polyadics defined as follows:

$$
\begin{aligned}
\tilde{\mathbf{\Lambda}}_{\alpha} & =\sum_{m=1}^{3} \frac{\hat{\mathbf{x}}_{m} \otimes \hat{\mathbf{x}}_{m}}{\Lambda_{\alpha}-\alpha_{m}^{2}}, \\
\tilde{\mathbf{\Lambda}}_{\alpha}^{\prime} & =\sum_{m=1}^{3} \frac{\hat{\mathbf{x}}_{m} \otimes \hat{\mathbf{x}}_{m}}{\Lambda_{\alpha}^{\prime}-\alpha_{m}^{2}}, \\
\tilde{\mathbf{H}}_{1}(\rho) & =\sum_{m=1}^{3} I_{1}^{m}(\rho) \hat{\mathbf{x}}_{m} \otimes \hat{\mathbf{x}}_{m}, \\
\tilde{\mathbf{H}}_{2}(\rho) & =\sum_{\substack{i, j=1 \\
i \neq j}}^{3} I_{2}^{i+j}(\rho) \hat{\mathbf{x}}_{i} \otimes \hat{\mathbf{x}}_{j} \otimes \hat{\mathbf{x}}_{i} \otimes \hat{\mathbf{x}}_{j}, \\
\tilde{\mathbf{F}}_{\alpha}(\mathbf{r}) & =-\frac{\mathbb{F}_{2}^{1}(\mathbf{r})}{\Lambda_{\alpha}-\Lambda_{\alpha}^{\prime}} \tilde{\mathbf{\Lambda}}_{\alpha}+\frac{\mathbb{F}_{2}^{2}(\mathbf{r})}{\Lambda_{\alpha}-\Lambda_{\alpha}^{\prime}} \tilde{\mathbf{\Lambda}}_{\alpha}^{\prime}+15 \mathbf{r} \otimes \mathbf{r}: \tilde{\tilde{\mathbf{H}}}_{2}(\rho) .
\end{aligned}
$$

The constants $\Lambda_{\alpha}, \Lambda_{\alpha}^{\prime}$ are given in (A.7) and the notation $\mathrm{O}\left(e l_{3}^{\prime}\right)$ indicates ellipsoidal terms in $\mathbf{r}^{\prime}$ that are of the octapole or higher multipole type.

A similar expansion for the potential $u_{\alpha}$ on $S_{\alpha}$ leads to

$$
u_{\alpha}\left(\alpha_{1}, \mu, \nu\right)=d_{0}^{1}+\sum_{m=1}^{3} \zeta_{1}^{m} E_{1}^{m}(\mu) E_{1}^{m}(\nu)+\sum_{m=1}^{5} \zeta_{2}^{m} E_{2}^{m}(\mu) E_{2}^{m}(\nu)+\mathrm{O}\left(e l_{3}^{\prime}\right)
$$


where for $n=1,2$ and $m=1,2, \ldots, 2 n+1$,

$$
\zeta_{n}^{m}=\frac{\mathbf{Q} \cdot \nabla \mathbb{E}_{n}^{m}\left(\mathbf{r}_{0}\right)}{\gamma_{n}^{m} C_{n}^{m}} \frac{1}{\alpha_{2} \alpha_{3} E_{n}^{m^{\prime}}\left(\alpha_{1}\right)} .
$$

Using the expression (2.21) as well as (A.3)-(A.19) we can rewrite the $\zeta_{n}^{m}$ for $n=1,2$ and $m=1,2, \ldots, 2 n+1$ as follows:

$$
\zeta_{1}^{m}=\frac{3 h_{m} \alpha_{m}}{4 \pi h_{1} h_{2} h_{3} \alpha_{1} \alpha_{2} \alpha_{3}} \cdot \frac{\mathbf{Q} \cdot \hat{\mathbf{x}}_{m}}{\sigma_{\alpha}+\left(\sigma_{b}-\sigma_{\alpha}\right)\left(\left(I_{n}^{m}\left(b_{1}\right)-I_{1}^{m}\left(\alpha_{1}\right)\right) b_{1} b_{2} b_{3}+\frac{b_{1} b_{2} b_{3}}{\alpha_{1} \alpha_{2} \alpha_{3}}\right)}
$$

for $m=1,2,3$,

$$
\begin{aligned}
\zeta_{2}^{1}= & -\frac{5}{8 \pi\left(\Lambda_{\alpha}-\Lambda_{\alpha}^{\prime}\right) \alpha_{1} \alpha_{2} \alpha_{3}} \\
& \times \frac{\mathbf{Q} \otimes \mathbf{r}_{0}: \tilde{\mathbf{\Lambda}}_{\alpha}}{\sigma_{\alpha}+\left(\sigma_{b}-\sigma_{\alpha}\right)\left(\left(I_{2}^{1}\left(b_{1}\right)-I_{2}^{1}\left(\alpha_{1}\right)\right) 2 \Lambda_{b} b_{1} b_{2} b_{3}+\frac{\Lambda_{b} b_{1} b_{2} b_{3}}{\Lambda_{\alpha} \alpha_{1} \alpha_{2} \alpha_{3}}\right)}, \\
\zeta_{2}^{2}= & \frac{5}{8 \pi\left(\Lambda_{\alpha}-\Lambda_{\alpha}^{\prime}\right) \alpha_{1} \alpha_{2} \alpha_{3}} \\
& \times \frac{\mathbf{Q} \otimes \mathbf{r}_{0}: \tilde{\mathbf{\Lambda}}_{\alpha}^{\prime}}{\sigma_{\alpha}+\left(\sigma_{b}-\sigma_{\alpha}\right)\left(\left(I_{2}^{2}\left(b_{1}\right)-I_{2}^{2}\left(\alpha_{1}\right)\right) 2 \Lambda_{b}^{\prime} b_{1} b_{2} b_{3}+\frac{\Lambda_{b}^{\prime} b_{1} b_{2} b_{3}}{\Lambda_{\alpha}^{\prime} \alpha_{1} \alpha_{2} \alpha_{3}}\right)}, \\
\zeta_{2}^{i+j}= & -\frac{15 \alpha_{i} \alpha_{j} h_{i} h_{j}}{4 \pi\left(h_{1} h_{2} h_{3}\right)^{2}\left(\alpha_{i}^{2}+\alpha_{j}^{2}\right) \alpha_{1} \alpha_{2} \alpha_{3}} \\
& \times \frac{\mathbf{Q} \cdot\left(x_{0 i} \hat{\mathbf{x}}_{j}+x_{0 j} \hat{\mathbf{x}}_{i}\right)}{\sigma_{\alpha}+\left(\sigma_{b}-\sigma_{\alpha}\right)\left(\left(I_{2}^{i+j}\left(b_{1}\right)-I_{2}^{i+j}\left(\alpha_{1}\right)\right)\left(b_{i}^{2}+b_{j}^{2}\right) b_{1} b_{2} b_{3}+\frac{\left(b_{i}^{2}+b_{j}^{2}\right) b_{1} b_{2} b_{3}}{\left(\alpha_{i}^{2}+\alpha_{j}^{2}\right) \alpha_{1} \alpha_{2} \alpha_{3}}\right)}
\end{aligned}
$$

with $i, j \in\{1,2,3\}$ and $i \neq j$.

Inserting (3.2) and (3.15) in (3.1) and using orthogonality we arrive at

$$
\mathbf{I}_{\alpha}(\mathbf{r})=\sum_{m=1}^{5} \zeta_{1}^{m} \gamma_{1}^{m} \boldsymbol{\beta}_{m}(\mathbf{r})+\sum_{m=1}^{5} \zeta_{2}^{m} \gamma_{2}^{m} \boldsymbol{\delta}_{m}(\mathbf{r})+\mathrm{O}\left(e l_{3}\right)
$$

or, in view of (3.3)-(3.14), (3.17)-(3.20) and some long calculations, we can rewrite 
(3.21) as

$$
\begin{aligned}
\mathbf{I}_{\alpha}(\mathbf{r})= & \sum_{m=1}^{3} \frac{3 \mathbf{Q} \cdot \hat{\mathbf{x}}_{m} \otimes \hat{\mathbf{x}}_{m} \otimes \mathbf{r} \times \tilde{\mathbf{H}}_{1}(\rho)}{\sigma_{\alpha}+\left(\sigma_{b}-\sigma_{\alpha}\right)\left(\left(I_{1}^{m}\left(b_{1}\right)-I_{1}^{m}\left(\alpha_{1}\right)\right) b_{1} b_{2} b_{3}+\frac{b_{1} b_{2} b_{3}}{\alpha_{1} \alpha_{2} \alpha_{3}}\right)} \\
& -\frac{\left(\Lambda_{\alpha}-\alpha_{1}^{2}\right)\left(\Lambda_{\alpha}-\alpha_{2}^{2}\right)\left(\Lambda_{\alpha}-\alpha_{3}^{2}\right) \mathbf{Q} \otimes \mathbf{r}_{0}: \tilde{\mathbf{\Lambda}}_{\alpha} \otimes \tilde{\mathbf{\Lambda}}_{\alpha} \times \tilde{\mathbf{F}}_{\alpha}(\mathbf{r})}{3\left(\Lambda_{\alpha}-\Lambda_{\alpha}^{\prime}\right)\left[\sigma_{\alpha}+\left(\sigma_{b}-\sigma_{\alpha}\right)\left(\left(I_{2}^{1}\left(b_{1}\right)-I_{2}^{1}\left(\alpha_{1}\right)\right) b_{1} b_{2} b_{3}+\frac{\Lambda_{b} b_{1} b_{2} b_{3}}{\Lambda_{\alpha} \alpha_{1} \alpha_{2} \alpha_{3}}\right)\right]} \\
& +\frac{\left(\Lambda_{\alpha}^{\prime}-\alpha_{1}^{2}\right)\left(\Lambda_{\alpha}^{\prime}-\alpha_{2}^{2}\right)\left(\Lambda_{\alpha}^{\prime}-\alpha_{3}^{2}\right) \mathbf{Q} \otimes \mathbf{r}_{0}: \tilde{\boldsymbol{\Lambda}}_{\alpha}^{\prime} \otimes \tilde{\mathbf{\Lambda}}_{\alpha}^{\prime} \times \tilde{\mathbf{F}}_{\alpha}(\mathbf{r})}{3\left(\Lambda_{\alpha}-\Lambda_{\alpha}^{\prime}\right)\left[\sigma_{\alpha}+\left(\sigma_{b}-\sigma_{\alpha}\right)\left(\left(I_{2}^{2}\left(b_{1}\right)-I_{2}^{2}\left(\alpha_{1}\right)\right) b_{1} b_{2} b_{3}+\frac{\Lambda_{b}^{\prime} b_{1} b_{2} b_{3}}{\Lambda_{\alpha}^{\prime} \alpha_{1} \alpha_{2} \alpha_{3}}\right)\right]} \\
& +\mathbf{Q} \otimes \mathbf{r}_{0}:\left[\sum_{\substack{i, j=1 \\
i \neq j}}^{3} \frac{\left(\hat{\mathbf{x}}_{i} \otimes \hat{\mathbf{x}}_{j}+\hat{\mathbf{x}}_{j} \otimes \hat{\mathbf{x}}_{i}\right) \otimes\left(\alpha_{i}^{2} \hat{\mathbf{x}}_{j} \otimes \hat{\mathbf{x}}_{i}+\alpha_{j}^{2} \hat{\mathbf{x}}_{i} \otimes \hat{\mathbf{x}}_{j}\right)}{\left(\alpha_{j}^{2}\right)}\right. \\
& \left.\left.\times \frac{\left(b_{i}^{2}+b_{j}^{2}\right) b_{1} b_{2} b_{3}}{\left(\alpha_{i}^{2}+\alpha_{j}^{2}\right) \alpha_{1} \alpha_{2} \alpha_{3}}\right)\right] \\
& \times \tilde{\mathbf{F}}_{\alpha}(\mathbf{r})+\mathrm{O}\left(e l_{3}\right)
\end{aligned}
$$

where the actual dependence on the position $\mathbf{r}_{0}$ and moment $\mathbf{Q}$ of the source dipole, on the observation point $\mathbf{r}$, on the geometrical parameters $\alpha_{i}, b_{i}, i=1,2,3$, and on the conductivities $\sigma_{\alpha}$ and $\sigma_{b}$, is explicitly shown.

Next we move to the integral

$$
\mathbf{I}_{b}(\mathbf{r})=\int_{S_{b}} u_{b}\left(\mathbf{r}^{\prime}\right) \hat{\boldsymbol{\rho}}^{\prime} \times \nabla_{\mathbf{r}^{\prime}} \frac{1}{\left|\mathbf{r}-\mathbf{r}^{\prime}\right|} d s\left(\mathbf{r}^{\prime}\right) .
$$

Following the same track of calculations as with the integral $\mathbf{I}_{\alpha}(\mathbf{r})$ we obtain

$$
\begin{aligned}
& \hat{\boldsymbol{\rho}}^{\prime} \times\left.\frac{\mathbf{r}-\mathbf{r}^{\prime}}{\left|\mathbf{r}-\mathbf{r}^{\prime}\right|^{3}}\right|_{\rho^{\prime}=b_{1}}=l_{b}\left(\mu^{\prime}, \nu^{\prime}\right)\left[\sum_{m=1}^{3} \boldsymbol{\beta}_{m}^{\prime} E_{1}^{m}\left(\mu^{\prime}\right) E_{1}^{m}\left(\nu^{\prime}\right)\right. \\
& \left.+\sum_{m=1}^{5} \boldsymbol{\delta}_{m}^{\prime} E_{2}^{m}\left(\mu^{\prime}\right) E_{2}^{m}\left(\nu^{\prime}\right)\right]+\mathrm{O}\left(e l_{3}^{\prime}\right) \\
& \boldsymbol{\beta}_{m}^{\prime}=3 \frac{b_{1} b_{2} b_{3}}{h_{1} h_{2} h_{3}} \frac{h_{m}}{b_{m}} \hat{\mathbf{x}}_{m} \otimes \mathbf{r} \times \tilde{\mathbf{H}}_{1}(\rho), \quad m=1,2,3, \\
& \boldsymbol{\delta}_{1}^{\prime}=-\frac{b_{1} b_{2} b_{3}}{3\left(\Lambda_{b}-\Lambda_{b}^{\prime}\right)} \tilde{\boldsymbol{\Lambda}}_{b} \times \tilde{\mathbf{F}}_{b}(\mathbf{r}), \\
& \boldsymbol{\delta}_{2}^{\prime}=\frac{b_{1} b_{2} b_{3}}{3\left(\Lambda_{b}-\Lambda_{b}^{\prime}\right)} \tilde{\mathbf{\Lambda}}_{b}^{\prime} \times \tilde{\mathbf{F}}_{b}(\mathbf{r}), \\
& \boldsymbol{\delta}_{3}^{\prime}=\frac{b_{1} b_{2} b_{3}}{h_{1} h_{2} h_{3}^{2}}\left[\frac{b_{2}}{b_{1}} \hat{\mathbf{x}}_{1} \otimes \hat{\mathbf{x}}_{2}+\frac{b_{1}}{b_{2}} \hat{\mathbf{x}}_{2} \otimes \hat{\mathbf{x}}_{1}\right] \times \tilde{\mathbf{F}}_{b}(\mathbf{r}),
\end{aligned}
$$




$$
\begin{aligned}
& \boldsymbol{\delta}_{4}^{\prime}=\frac{b_{1} b_{2} b_{3}}{h_{1} h_{2}^{2} h_{3}}\left[\frac{b_{3}}{b_{1}} \hat{\mathbf{x}}_{1} \otimes \hat{\mathbf{x}}_{3}+\frac{b_{1}}{b_{3}} \hat{\mathbf{x}}_{3} \otimes \hat{\mathbf{x}}_{1}\right] \times \tilde{\mathbf{F}}_{b}(\mathbf{r}), \\
& \boldsymbol{\delta}_{5}^{\prime}=\frac{b_{1} b_{2} b_{3}}{h_{1}^{2} h_{2} h_{3}}\left[\frac{b_{3}}{b_{2}} \hat{\mathbf{x}}_{2} \otimes \hat{\mathbf{x}}_{3}+\frac{b_{2}}{b_{3}} \hat{\mathbf{x}}_{3} \otimes \hat{\mathbf{x}}_{2}\right] \times \tilde{\mathbf{F}}_{b}(\mathbf{r}),
\end{aligned}
$$

where $\Lambda_{b}, \Lambda_{b}^{\prime}$ and $l_{b}\left(\mu^{\prime}, \nu^{\prime}\right)$ are given by (A.7) and (A.14) respectively, with $\alpha_{i}$ replaced by $b_{i}, i=1,2,3$,

$$
\left.\begin{array}{l}
\tilde{\mathbf{\Lambda}}_{b}=\sum_{m=1}^{3} \frac{\hat{\mathbf{x}}_{m} \otimes \hat{\mathbf{x}}_{m}}{\Lambda_{b}-b_{m}^{2}} \\
\tilde{\boldsymbol{\Lambda}}_{b}^{\prime}=\sum_{m=1}^{3} \frac{\hat{\mathbf{x}}_{m} \otimes \hat{\mathbf{x}}_{m}}{\Lambda_{b}^{\prime}-b_{m}^{2}}
\end{array}\right\}
$$

and

$$
\tilde{\mathbf{F}}_{b}(\mathbf{r})=-\frac{\mathbb{F}_{2}^{1}(\mathbf{r})}{\Lambda_{b}-\Lambda_{b}^{\prime}} \tilde{\mathbf{\Lambda}}_{b}+\frac{\mathbb{F}_{2}^{2}(\mathbf{r})}{\Lambda_{b}-\Lambda_{b}^{\prime}} \tilde{\mathbf{\Lambda}}_{b}^{\prime}+15 \mathbf{r} \otimes \mathbf{r}: \tilde{\tilde{\mathbf{H}}}_{2}(\rho)
$$

The dyadic function $\tilde{\mathbf{H}}_{1}(\rho)$ and the tetradic function $\tilde{\tilde{\mathbf{H}}}_{2}(\rho)$ are defined by $(\underline{3.12})$ and (3.13) respectively.

It is straightforward to show that as a consequence of confocality

$$
\left.\begin{array}{c}
\Lambda_{\alpha}-\Lambda_{\alpha}^{\prime}=\Lambda_{b}-\Lambda_{b}^{\prime} \\
\Lambda_{\alpha}-\alpha_{i}^{2}=\Lambda_{b}-b_{i}^{2}, \quad i=1,2,3 \\
\Lambda_{\alpha}^{\prime}-\alpha_{i}^{2}=\Lambda_{b}^{\prime}-b_{i}^{2}, \quad i=1,2,3
\end{array}\right\}
$$

and

$$
\left.\begin{array}{l}
\tilde{\mathbf{\Lambda}}_{\alpha}=\tilde{\boldsymbol{\Lambda}}_{b}=\tilde{\boldsymbol{\Lambda}} \\
\tilde{\mathbf{\Lambda}}_{\alpha}^{\prime}=\tilde{\boldsymbol{\Lambda}}_{b}^{\prime}=\tilde{\boldsymbol{\Lambda}}^{\prime}
\end{array}\right\}
$$

Hence

$$
\tilde{\mathbf{F}}_{\alpha}(\mathbf{r})=\tilde{\mathbf{F}}_{b}(\mathbf{r})=\tilde{\mathbf{F}}(\mathbf{r}) .
$$

The potential (2.20) provides

$$
u_{b}\left(b_{1}, \mu, \nu\right)=d_{0}^{1}+\sum_{m=1}^{3} \theta_{1}^{m} E_{1}^{m}(\mu) E_{1}^{m}(\nu)+\sum_{m=1}^{5} \theta_{2}^{m} E_{2}^{m}(\mu) E_{2}^{m}(\nu)+\mathrm{O}\left(e l_{3}\right)
$$

where for $n=1,2$ and $m=1,2, \ldots, 2 n+1$,

$$
\theta_{n}^{m}=\frac{\mathbf{Q} \cdot \nabla \mathbb{E}_{n}^{m}\left(\mathbf{r}_{0}\right) E_{n}^{m}\left(b_{1}\right)}{\gamma_{n}^{m} C_{n}^{m}}\left(I_{n}^{m}\left(b_{1}\right)-I_{n}^{m}\left(\alpha_{1}\right)+\frac{1}{E_{n}^{m}\left(\alpha_{1}\right) E_{n}^{m^{\prime}}\left(\alpha_{1}\right) \alpha_{2} \alpha_{3}}\right) .
$$

Further manipulations imply that

$$
\theta_{1}^{m}=\frac{3 h_{m} b_{m}}{4 \pi h_{1} h_{2} h_{3}} \cdot \frac{\mathbf{Q} \cdot \hat{\mathbf{x}}_{m}\left(I_{1}^{m}\left(b_{1}\right)-I_{1}^{m}\left(\alpha_{1}\right)+\frac{1}{\alpha_{1} \alpha_{2} \alpha_{3}}\right)}{\sigma_{\alpha}+\left(\sigma_{b}-\sigma_{\alpha}\right)\left(\left(I_{1}^{m}\left(b_{1}\right)-I_{1}^{m}\left(\alpha_{1}\right)\right) b_{1} b_{2} b_{3}+\frac{b_{1} b_{2} b_{3}}{\alpha_{1} \alpha_{2} \alpha_{3}}\right)}
$$


for $m=1,2,3$,

$$
\begin{aligned}
\theta_{2}^{1}= & -\frac{5 \mathbf{Q} \otimes \mathbf{r}_{0}: \tilde{\mathbf{\Lambda}}}{8 \pi\left(\Lambda_{b}-\Lambda_{b}^{\prime}\right)} \cdot \frac{\left(I_{2}^{1}\left(b_{1}\right)-I_{2}^{1}\left(\alpha_{1}\right)\right) 2 \Lambda_{b}+\frac{\Lambda_{b}}{\Lambda_{\alpha} \alpha_{1} \alpha_{2} \alpha_{3}}}{\sigma_{\alpha}+\left(\sigma_{b}-\sigma_{\alpha}\right)\left(\left(I_{2}^{1}\left(b_{1}\right)-I_{2}^{1}\left(\alpha_{1}\right)\right) 2 \Lambda_{b} b_{1} b_{2} b_{3}+\frac{\Lambda_{b} b_{1} b_{2} b_{3}}{\Lambda_{\alpha} \alpha_{1} \alpha_{2} \alpha_{3}}\right)}, \\
\theta_{2}^{2}= & \frac{5 \mathbf{Q} \otimes \mathbf{r}_{0}: \tilde{\mathbf{\Lambda}}^{\prime}}{8 \pi\left(\Lambda_{b}-\Lambda_{b}^{\prime}\right)} \cdot \frac{\left(I_{2}^{2}\left(b_{1}\right)-I_{2}^{2}\left(\alpha_{1}\right)\right) 2 \Lambda_{b}^{\prime}+\frac{\Lambda_{b}^{\prime}}{\Lambda_{\alpha}^{\prime} \alpha_{1} \alpha_{2} \alpha_{3}}}{\sigma_{\alpha}+\left(\sigma_{b}-\sigma_{\alpha}\right)\left(\left(I_{2}^{2}\left(b_{1}\right)-I_{2}^{2}\left(\alpha_{1}\right)\right) 2 \Lambda_{b}^{\prime} b_{1} b_{2} b_{3}+\frac{\Lambda_{b}^{\prime} b_{1} b_{2} b_{3}}{\Lambda_{\alpha}^{\prime} \alpha_{1} \alpha_{2} \alpha_{3}}\right)}, \\
& \theta_{2}^{i+j}=\frac{15 \mathbf{Q} \cdot\left(x_{0 i} \hat{\mathbf{x}}_{j}+x_{0 j} \hat{\mathbf{x}}_{i}\right) h_{i} h_{j} b_{i} b_{j}}{4 \pi h_{1}^{2} h_{2}^{2} h_{3}^{2}} \\
& \times \frac{I_{2}^{i+j}\left(b_{1}\right)-I_{2}^{i+j}\left(\alpha_{1}\right)+\frac{1}{\left(\alpha_{i}^{2}+\alpha_{j}^{2}\right) \alpha_{1} \alpha_{2} \alpha_{3}}}{\sigma_{\alpha}+\left(\sigma_{b}-\sigma_{\alpha}\right)\left(\left(I_{2}^{i+j}\left(b_{1}\right)-I_{2}^{i+j}\left(\alpha_{1}\right)\right)\left(b_{i}^{2}+b_{j}^{2}\right) b_{1} b_{2} b_{3}+\frac{\left(b_{i}^{2}+b_{j}^{2}\right) b_{1} b_{2} b_{3}}{\left(\alpha_{i}^{2}+\alpha_{j}^{2}\right) \alpha_{1} \alpha_{2} \alpha_{3}}\right)}
\end{aligned}
$$

with $i, j=\{1,2,3\}$ and $i \neq j$.

Using orthogonality, the integral (3.23), with the help of the multipole expansions (3.24) and (3.37), provides

$$
\mathbf{I}_{b}(\mathbf{r})=\sum_{m=1}^{3} \theta_{1}^{m} \gamma_{1}^{m} \boldsymbol{\beta}_{m}^{\prime}(\mathbf{r})+\sum_{m=1}^{5} \theta_{2}^{m} \gamma_{2}^{m} \boldsymbol{\delta}_{m}^{\prime}(\mathbf{r})+\mathrm{O}\left(e l_{3}\right) .
$$

Using (3.25)-(3.30) and (3.39)-(3.42), as well as a long series of calculations, it is possible to rewrite (3.43) as

$$
\begin{aligned}
\mathbf{I}_{b}(\mathbf{r})= & \sum_{m=1}^{3} \frac{\left(I_{1}^{m}\left(b_{1}\right)-I_{1}^{m}\left(\alpha_{1}\right)\right) b_{1} b_{2} b_{3}+\frac{b_{1} b_{2} b_{3}}{\alpha_{1} \alpha_{2} \alpha_{3}}}{\sigma_{\alpha}+\left(\sigma_{b}-\sigma_{\alpha}\right)\left(\left(I_{1}^{m}\left(b_{1}\right)-I_{1}^{m}\left(\alpha_{1}\right)\right) b_{1} b_{2} b_{3}+\frac{b_{1} b_{2} b_{3}}{\alpha_{1} \alpha_{2} \alpha_{3}}\right)} \\
& \times 3 \mathbf{Q} \cdot \hat{\mathbf{x}}_{m} \otimes \hat{\mathbf{x}}_{m} \otimes \mathbf{r} \times \tilde{\mathbf{H}}_{1}(\rho) \\
& -\frac{\left(\Lambda_{\alpha}-\alpha_{1}^{2}\right)\left(\Lambda_{\alpha}-\alpha_{2}^{2}\right)\left(\Lambda_{\alpha}-\alpha_{3}^{2}\right)\left(\left(I_{2}^{1}\left(b_{1}\right)-I_{2}^{1}\left(\alpha_{1}\right)\right) 2 \Lambda_{b} b_{1} b_{2} b_{3}+\frac{\Lambda_{b} b_{1} b_{2} b_{3}}{\Lambda_{\alpha} \alpha_{1} \alpha_{2} \alpha_{3}}\right)}{3\left(\Lambda_{\alpha}-\Lambda_{\alpha}^{\prime}\right)\left[\sigma_{\alpha}+\left(\sigma_{b}-\sigma_{\alpha}\right)\left(\left(I_{2}^{1}\left(b_{1}\right)-I_{2}^{1}\left(\alpha_{1}\right)\right) 2 \Lambda_{b} b_{1} b_{2} b_{3}+\frac{\Lambda_{b} b_{1} b_{2} b_{3}}{\Lambda_{\alpha} \alpha_{1} \alpha_{2} \alpha_{3}}\right)\right]} \\
& \times \mathbf{Q} \otimes \mathbf{r}_{0}: \tilde{\mathbf{\Lambda}} \otimes \tilde{\mathbf{\Lambda}} \times \mathbf{F}(\mathbf{r}) \\
& +\frac{\left(\Lambda_{\alpha}^{\prime}-\alpha_{1}^{2}\right)\left(\Lambda_{\alpha}^{\prime}-\alpha_{2}^{2}\right)\left(\Lambda_{\alpha}^{\prime}-\alpha_{3}^{2}\right)\left(\left(I_{2}^{2}\left(b_{1}\right)-I_{2}^{2}\left(\alpha_{1}\right)\right) 2 \Lambda_{b}^{\prime} b_{1} b_{2} b_{3}+\frac{\Lambda_{b}^{\prime} b_{1} b_{2} b_{3}}{\Lambda_{\alpha}^{\prime} \alpha_{1} \alpha_{2} \alpha_{3}}\right)}{3\left(\Lambda_{\alpha}-\Lambda_{\alpha}^{\prime}\right)\left[\sigma_{\alpha}+\left(\sigma_{b}-\sigma_{\alpha}\right)\left(\left(I_{2}^{2}\left(b_{1}\right)-I_{2}^{2}\left(\alpha_{1}\right)\right) 2 \Lambda_{b}^{\prime} b_{1} b_{2} b_{3}+\frac{\Lambda_{b}^{\prime} b_{1} b_{2} b_{3}}{\Lambda_{\alpha}^{\prime} \alpha_{1} \alpha_{2} \alpha_{3}}\right)\right]} \\
& \times \mathbf{Q} \otimes \mathbf{r}_{0}: \tilde{\mathbf{\Lambda}}^{\prime} \otimes \tilde{\mathbf{\Lambda}}^{\prime} \times \mathbf{F}(\mathbf{r}) \\
& +\sum_{i, j=1}^{3} \frac{\left(I_{2}^{i+j}\left(b_{1}\right)-I_{2}^{i+j}\left(\alpha_{1}\right)\right)\left(b_{i}^{2}+b_{j}^{2}\right) b_{1} b_{2} b_{3}+\frac{\left(b_{i}^{2}+b_{j}^{2}\right) b_{1} b_{2} b_{3}}{\left(\alpha_{i}^{2}+\alpha_{j}^{2}\right) \alpha_{1} \alpha_{2} \alpha_{3}}}{\sigma_{\alpha}+\left(\sigma_{b}-\sigma_{\alpha}\right)\left(\left(I_{2}^{i+j}\left(b_{1}\right)-I_{2}^{i+j}\left(\alpha_{1}\right)\right)\left(b_{i}^{2}+b_{j}^{2}\right) b_{1} b_{2} b_{3}+\frac{\left(b_{i}^{2}+b_{j}^{2}\right) b_{1} b_{2} b_{3}}{\left(\alpha_{i}^{2}+\alpha_{j}^{2}\right) \alpha_{1} \alpha_{2} \alpha_{3}}\right)} \\
& \times \frac{\mathbf{Q} \otimes \mathbf{r}_{0} \cdot\left(\hat{\mathbf{x}}_{i} \otimes \hat{\mathbf{x}}_{j}+\hat{\mathbf{x}}_{j} \otimes \hat{\mathbf{x}}_{i}\right) \otimes\left(b_{i}^{2} \hat{\mathbf{x}}_{j} \otimes \hat{\mathbf{x}}_{i}+b_{j}^{2} \hat{\mathbf{x}}_{i} \otimes \hat{\mathbf{x}}_{j}\right) \times \tilde{\mathbf{F}}(\mathbf{r})}{b_{i}^{2}+b_{j}^{2}} .
\end{aligned}
$$



[3]

The multipole expansion of the first term on the right-hand side of (2.9) implies that

$$
\mathbf{Q} \times \frac{\mathbf{r}-\mathbf{r}_{0}}{\left|\mathbf{r}-\mathbf{r}_{0}\right|^{3}}=3 \mathbf{Q} \times \tilde{\mathbf{H}}_{1}(\rho) \cdot \mathbf{r}+\mathbf{Q} \times \mathbf{r}_{0} \cdot \tilde{\mathbf{F}}(\mathbf{r})+O\left(e l_{3}\right)
$$

At this stage we have the multipole expansions of all terms appearing in (2.9), which by virtue of the continuity condition (2.15) is written as

$$
\mathbf{B}(\mathbf{r})=\frac{\mu_{0}}{4 \pi}\left[\mathbf{Q} \times \frac{\mathbf{r}-\mathbf{r}_{0}}{\left|\mathbf{r}-\mathbf{r}_{0}\right|^{3}}-\sigma_{\alpha} \mathbf{I}_{\alpha}(\mathbf{r})-\left(\sigma_{b}-\sigma_{\alpha}\right) \mathbf{I}_{b}(\mathbf{r})\right]
$$

First we observe that the dipole term of the expression involving $\mathbf{I}_{\alpha}(\mathbf{r})$ and $\mathbf{I}_{b}(\mathbf{r})$ gives

$$
\begin{aligned}
& {\left[\sigma_{\alpha} \mathbf{I}_{\alpha}(\mathbf{r})+\left(\sigma_{b}-\sigma_{\alpha}\right) \mathbf{I}_{b}(\mathbf{r})\right]_{n=1}=\sigma_{\alpha} \sum_{m=1}^{3} \zeta_{1}^{m} \boldsymbol{\beta}_{m}(\mathbf{r}) \gamma_{1}^{m}+\left(\sigma_{b}-\sigma_{\alpha}\right) \sum_{m=1}^{3} \theta_{1}^{m} \boldsymbol{\beta}_{m}^{\prime}(\mathbf{r}) \gamma_{1}^{m} } \\
= & \sum_{m=1}^{3} \frac{3 \sigma_{\alpha} \mathbf{Q} \cdot \hat{\mathbf{x}}_{m} \otimes \hat{\mathbf{x}}_{m} \otimes \mathbf{r} \times \tilde{\mathbf{H}}_{1}(\rho)}{\sigma_{\alpha}+\left(\sigma_{b}-\sigma_{\alpha}\right)\left(\left(I_{1}^{m}\left(b_{1}\right)-I_{1}^{m}\left(\alpha_{1}\right)\right) b_{1} b_{2} b_{3}+\frac{b_{1} b_{2} b_{3}}{\alpha_{1} \alpha_{2} \alpha_{3}}\right)} \\
& +\frac{\left(\sigma_{b}-\sigma_{\alpha}\right)\left(\left(I_{1}^{m}\left(b_{1}\right)-I_{1}^{m}\left(\alpha_{1}\right)\right) b_{1} b_{2} b_{3}+\frac{b_{1} b_{2} b_{3}}{\alpha_{1} \alpha_{2} \alpha_{3}}\right)}{\sigma_{\alpha}+\left(\sigma_{b}-\sigma_{\alpha}\right)\left(\left(I_{1}^{m}\left(b_{1}\right)-I_{1}^{m}\left(\alpha_{1}\right)\right) b_{1} b_{2} b_{3}+\frac{b_{1} b_{2} b_{3}}{\alpha_{1} \alpha_{2} \alpha_{3}}\right)} 3 \mathbf{Q} \cdot \hat{\mathbf{x}}_{m} \otimes \hat{\mathbf{x}}_{m} \otimes \mathbf{r} \times \tilde{\mathbf{H}}_{1}(\rho) \\
= & 3 \mathbf{Q} \cdot \sum_{m=1}^{3} \hat{\mathbf{x}}_{m} \otimes \hat{\mathbf{x}}_{m} \otimes \mathbf{r} \times \tilde{\mathbf{H}}_{1}(\rho)=3 \mathbf{Q} \cdot \tilde{\mathbf{I}} \otimes \mathbf{r} \times \tilde{\mathbf{H}}_{1}(\rho)=3 \mathbf{Q} \otimes \mathbf{r} \times \tilde{\mathbf{H}}_{1}(\rho)
\end{aligned}
$$

where $\tilde{\mathbf{I}}$ stands for the identity dyadic. Combining this result with (3.45) we see that the dipole contribution to $\mathbf{B}$ vanishes. This result is in accord with the physical fact that no magnetic monopole exists in nature. Consequently, the leading multipole of $\mathbf{B}$ is of quadrupolic character.

Analyzing each one of the five types of quadrupoles appearing in the part of $\mathbf{B}$ that is due to the conductive current we obtain

$$
\begin{aligned}
& {\left[\sigma_{\alpha} \mathbf{I}_{\alpha}(\mathbf{r})+\left(\sigma_{b}-\sigma_{\alpha}\right) \mathbf{I}_{b}(\mathbf{r})\right]_{2}^{1}} \\
& \quad=-\frac{\left(\Lambda_{\alpha}-\alpha_{1}^{2}\right)\left(\Lambda_{\alpha}-\alpha_{2}^{2}\right)\left(\Lambda_{\alpha}-\alpha_{3}^{2}\right)}{3\left(\Lambda_{\alpha}-\Lambda_{\alpha}^{\prime}\right)} \mathbf{Q} \otimes \mathbf{r}_{0}: \tilde{\mathbf{\Lambda}} \otimes \tilde{\mathbf{\Lambda}} \times \tilde{\mathbf{F}}(\mathbf{r}), \\
& {\left[\sigma_{\alpha} \mathbf{I}_{\alpha}(\mathbf{r})+\left(\sigma_{b}-\sigma_{\alpha}\right) \mathbf{I}_{b}(\mathbf{r})\right]_{2}^{2}} \\
& =\frac{\left(\Lambda_{\alpha}^{\prime}-\alpha_{1}^{2}\right)\left(\Lambda_{\alpha}^{\prime}-\alpha_{2}^{2}\right)\left(\Lambda_{\alpha}^{\prime}-\alpha_{3}^{2}\right)}{3\left(\Lambda_{\alpha}-\Lambda_{\alpha}^{\prime}\right)} \mathbf{Q} \otimes \mathbf{r}_{0}: \tilde{\mathbf{\Lambda}}^{\prime} \otimes \tilde{\mathbf{\Lambda}}^{\prime} \times \tilde{\mathbf{F}}(\mathbf{r}),
\end{aligned}
$$


and for $i, j=\{1,2,3\}$ with $i \neq j$,

$$
\begin{aligned}
& {\left[\sigma_{\alpha} \mathbf{I}_{\alpha}(\mathbf{r})+\left(\sigma_{b}-\sigma_{\alpha}\right) \mathbf{I}_{b}(\mathbf{r})\right]_{2}^{i+j}} \\
& =\mathbf{Q} \otimes \mathbf{r}_{0}: \frac{\left(\hat{\mathbf{x}}_{i} \otimes \hat{\mathbf{x}}_{j}+\hat{\mathbf{x}}_{j} \otimes \hat{\mathbf{x}}_{i}\right) \otimes\left(b_{i}^{2} \hat{\mathbf{x}}_{j} \otimes \hat{\mathbf{x}}_{i}+b_{j}^{2} \hat{\mathbf{x}}_{i} \otimes \hat{\mathbf{x}}_{j}\right)}{b_{i}^{2}+b_{j}^{2}} \times \tilde{\mathbf{F}}(\mathbf{r}) \\
& +\frac{\sigma_{\alpha} \mathbf{Q} \otimes \mathbf{r}_{0}:\left(\hat{\mathbf{x}}_{i} \otimes \hat{\mathbf{x}}_{j}+\hat{\mathbf{x}}_{j} \otimes \hat{\mathbf{x}}_{i}\right)}{\sigma_{\alpha}+\left(\sigma_{b}-\sigma_{\alpha}\right)\left(\left(I_{2}^{i+j}\left(b_{1}\right)-I_{2}^{i+j}\left(\alpha_{1}\right)\right)\left(b_{i}^{2}+b_{j}^{2}\right)+\frac{\left(b_{i}^{2}+b_{j}^{2}\right) b_{1} b_{2} b_{3}}{\left.\left(\alpha_{i}^{2}+\alpha_{j}^{2}\right) \alpha_{1} \alpha_{2} \alpha_{3}\right)}\right.} \\
& \otimes\left(\frac{\alpha_{i}^{2} \hat{\mathbf{x}}_{j} \otimes \hat{\mathbf{x}}_{i}+\alpha_{j}^{2} \hat{\mathbf{x}}_{i} \otimes \hat{\mathbf{x}}_{j}}{\alpha_{i}^{2}+\alpha_{j}^{2}}-\frac{b_{i}^{2} \hat{\mathbf{x}}_{j} \otimes \hat{\mathbf{x}}_{i}+b_{j}^{2} \hat{\mathbf{x}}_{i} \otimes \hat{\mathbf{x}}_{j}}{b_{i}^{2}+b_{j}^{2}}\right) \times \tilde{\mathbf{F}}(\mathbf{r}) .
\end{aligned}
$$

Substituting (3.48)-(3.50) in (3.46) and using the identities

$$
\begin{aligned}
& \frac{\left(\Lambda_{\alpha}-\alpha_{1}^{2}\right)\left(\Lambda_{\alpha}-\alpha_{2}^{2}\right)\left(\Lambda_{\alpha}-\alpha_{3}^{2}\right)}{3\left(\Lambda_{\alpha}-\Lambda_{\alpha}^{\prime}\right)} \tilde{\mathbf{\Lambda}} \otimes \tilde{\mathbf{\Lambda}} \\
& -\frac{\left(\Lambda_{\alpha}^{\prime}-\alpha_{1}^{2}\right)\left(\Lambda_{\alpha}^{\prime}-\alpha_{2}^{2}\right)\left(\Lambda_{\alpha}^{\prime}-\alpha_{3}^{2}\right)}{3\left(\Lambda_{\alpha}-\Lambda_{\alpha}^{\prime}\right)} \tilde{\Lambda}^{\prime} \otimes \tilde{\boldsymbol{\Lambda}}^{\prime} \\
& =\frac{1}{3} \tilde{\mathbf{I}} \otimes \tilde{\mathbf{I}}-\sum_{i=1}^{3} \hat{\mathbf{x}}_{i} \otimes \hat{\mathbf{x}}_{i} \otimes \hat{\mathbf{x}}_{i} \otimes \hat{\mathbf{x}}_{i}, \\
& \frac{\left(\hat{\mathbf{x}}_{i} \otimes \hat{\mathbf{x}}_{j}+\hat{\mathbf{x}}_{j} \otimes \hat{\mathbf{x}}_{i}\right) \otimes\left(b_{j}^{2} \hat{\mathbf{x}}_{i} \otimes \hat{\mathbf{x}}_{j}+b_{i}^{2} \hat{\mathbf{x}}_{j} \otimes \hat{\mathbf{x}}_{i}\right)}{b_{i}^{2}+b_{j}^{2}} \\
& =\hat{\mathbf{x}}_{i} \otimes \hat{\mathbf{x}}_{j} \otimes \hat{\mathbf{x}}_{i} \otimes \hat{\mathbf{x}}_{j} \\
& +\hat{\mathbf{x}}_{j} \otimes \hat{\mathbf{x}}_{i} \otimes \hat{\mathbf{x}}_{j} \otimes \hat{\mathbf{x}}_{i}+\frac{\left(b_{i}^{2} \hat{\mathbf{x}}_{i} \otimes \hat{\mathbf{x}}_{j}-b_{j}^{2} \hat{\mathbf{x}}_{j} \otimes \hat{\mathbf{x}}_{i}\right) \otimes\left(\hat{\mathbf{x}}_{j} \otimes \hat{\mathbf{x}}_{i}-\hat{\mathbf{x}}_{i} \otimes \hat{\mathbf{x}}_{j}\right)}{b_{i}^{2}+b_{j}^{2}}, \quad i \neq j, \\
& \hat{\mathbf{x}}_{1} \times \tilde{\mathbf{I}}=\hat{\mathbf{x}}_{3} \otimes \hat{\mathbf{x}}_{2}-\hat{\mathbf{x}}_{2} \otimes \hat{\mathbf{x}}_{3} \\
& \left.\hat{\mathbf{x}}_{2} \times \tilde{\mathbf{I}}=\hat{\mathbf{x}}_{1} \otimes \hat{\mathbf{x}}_{3}-\hat{\mathbf{x}}_{3} \otimes \hat{\mathbf{x}}_{1}\right\}, \\
& \left.\hat{\mathbf{x}}_{3} \times \tilde{\mathbf{I}}=\hat{\mathbf{x}}_{2} \otimes \hat{\mathbf{x}}_{1}-\hat{\mathbf{x}}_{1} \otimes \hat{\mathbf{x}}_{2}\right\} \\
& \sum_{\substack{(i, j)=\{(1,2) \\
(1,3) \\
(2,3)\}}}^{3} \frac{\left(\hat{\mathbf{x}}_{i} \otimes \hat{\mathbf{x}}_{j}+\hat{\mathbf{x}}_{j} \otimes \hat{\mathbf{x}}_{i}\right) \otimes\left(b_{j}^{2} \hat{\mathbf{x}}_{i} \otimes \hat{\mathbf{x}}_{j}+b_{i}^{2} \hat{\mathbf{x}}_{j} \otimes \hat{\mathbf{x}}_{i}\right)}{b_{i}^{2}+b_{j}^{2}} \\
& =\sum_{\substack{i, j=1 \\
i \neq j}}^{3} \hat{\mathbf{x}}_{i} \otimes \hat{\mathbf{x}}_{j} \otimes \hat{\mathbf{x}}_{i} \otimes \hat{\mathbf{x}}_{j} \\
& +\left\{\frac{\left(b_{1}^{2} \hat{\mathbf{x}}_{1} \otimes \hat{\mathbf{x}}_{2}-b_{2}^{2} \hat{\mathbf{x}}_{2} \otimes \hat{\mathbf{x}}_{1}\right) \otimes \hat{\mathbf{x}}_{3}}{b_{1}^{2}+b_{2}^{2}}+\frac{\left(-b_{1}^{2} \hat{\mathbf{x}}_{1} \otimes \hat{\mathbf{x}}_{3}+b_{3}^{2} \hat{\mathbf{x}}_{3} \otimes \hat{\mathbf{x}}_{1}\right) \otimes \hat{\mathbf{x}}_{2}}{b_{1}^{2}+b_{3}^{2}}\right. \\
& \left.+\frac{\left(b_{2}^{2} \hat{\mathbf{x}}_{2} \otimes \hat{\mathbf{x}}_{3}-b_{3}^{2} \hat{\mathbf{x}}_{3} \otimes \hat{\mathbf{x}}_{2}\right) \otimes \hat{\mathbf{x}}_{1}}{b_{2}^{2}+b_{3}^{2}}\right\} \times \tilde{\mathbf{I}},
\end{aligned}
$$




$$
\begin{gathered}
\mathbf{Q} \otimes \mathbf{r}_{0}:\left(\sum_{i=1}^{3} \hat{\mathbf{x}}_{i} \otimes \hat{\mathbf{x}}_{i} \otimes \hat{\mathbf{x}}_{i} \otimes \hat{\mathbf{x}}_{i}+\sum_{\substack{i, j=1 \\
i \neq j}}^{3} \hat{\mathbf{x}}_{i} \otimes \hat{\mathbf{x}}_{j} \otimes \hat{\mathbf{x}}_{i} \otimes \hat{\mathbf{x}}_{j}\right) \times \tilde{\mathbf{F}}(\mathbf{r}) \\
=\mathbf{Q} \otimes \mathbf{r}_{0}: \tilde{\tilde{\mathbf{I}}} \times \tilde{\mathbf{F}}(\mathbf{r})=\mathbf{Q} \otimes \mathbf{r}_{0} \underset{.}{\mathbf{F}}(\mathbf{r})=\mathbf{Q} \times \mathbf{r}_{0} \cdot \tilde{\mathbf{F}}(\mathbf{r})
\end{gathered}
$$

and

$$
\tilde{\mathbf{I}} \times \tilde{\mathbf{F}}(\mathbf{r})=\tilde{\mathbf{O}}
$$

where $\tilde{\tilde{\mathbf{I}}}$ is the identity tetradic defined by

$$
\tilde{\tilde{\mathbf{I}}}=\sum_{i, j=1}^{3} \hat{\mathbf{x}}_{i} \otimes \hat{\mathbf{x}}_{j} \otimes \hat{\mathbf{x}}_{i} \otimes \hat{\mathbf{x}}_{j}
$$

we arrive at the compact expression

$$
\begin{aligned}
\mathbf{B}(\mathbf{r})= & \frac{\mu_{0}}{4 \pi}\left(\mathbf{d}-\mathbf{d}_{b}+\mathbf{d}_{\alpha}\right) \\
& \left(\frac{\mathbb{F}_{2}^{1}(\mathbf{r})}{\Lambda_{\alpha}-\Lambda_{\alpha}^{\prime}} \tilde{\mathbf{\Lambda}}-\frac{\mathbb{F}_{2}^{2}(\mathbf{r})}{\Lambda_{\alpha}-\Lambda_{\alpha}^{\prime}} \tilde{\mathbf{\Lambda}}^{\prime}-15 \sum_{\substack{i, j=1 \\
i \neq j}}^{3} x_{i} x_{j} I_{2}^{i+j}(\rho) \hat{\mathbf{x}}_{i} \otimes \hat{\mathbf{x}}_{j}\right)+\mathrm{O}\left(e l_{3}\right)
\end{aligned}
$$

where

$$
\begin{aligned}
\mathbf{d} & =\left(\mathbf{Q} \cdot \tilde{\mathbf{M}}\left(b_{1}\right) \times \mathbf{r}_{0}\right) \cdot \tilde{\mathbf{N}}\left(b_{1}\right), \\
\mathbf{d}_{b} & =\left(\mathbf{Q} \cdot \tilde{\mathbf{M}}\left(b_{1}\right) \times \mathbf{r}_{0}\right) \cdot \tilde{\mathbf{N}}_{c}\left(b_{1}\right), \\
\mathbf{d}_{\alpha} & =\left(\mathbf{Q} \cdot \tilde{\mathbf{M}}\left(\alpha_{1}\right) \times \mathbf{r}_{0}\right) \cdot \tilde{\mathbf{N}}_{c}\left(\alpha_{1}\right), \\
\tilde{\mathbf{M}}\left(b_{1}\right) & =\sum_{i=1}^{3} b_{i}^{2} \hat{\mathbf{x}}_{i} \otimes \hat{\mathbf{x}}_{i}, \\
\tilde{\mathbf{M}}\left(\alpha_{1}\right) & =\sum_{i=1}^{3} \alpha_{i}^{2} \hat{\mathbf{x}}_{i} \otimes \hat{\mathbf{x}}_{i}, \\
\tilde{\mathbf{N}}\left(b_{1}\right) & =\sum_{i=1}^{3} \frac{\hat{\mathbf{x}}_{i} \otimes \hat{\mathbf{x}}_{i}}{b_{1}^{2}+b_{2}^{2}+b_{3}^{2}-b_{i}^{2}}, \\
\tilde{\mathbf{N}}_{c}\left(b_{1}\right) & =\sum_{i=1}^{3} C^{6-i} \frac{\hat{\mathbf{x}}_{i} \otimes \hat{\mathbf{x}}_{i}}{b_{1}^{2}+b_{2}^{2}+b_{3}^{2}-b_{i}^{2}}, \\
\tilde{\mathbf{N}}_{c}\left(\alpha_{1}\right) & =\sum_{i=1}^{3} C^{6-i} \frac{\hat{\mathbf{x}}_{i} \otimes \hat{\mathbf{x}}_{i}}{\alpha_{1}^{2}+\alpha_{2}^{2}+\alpha_{3}^{2}-\alpha_{i}^{2}}
\end{aligned}
$$


and

$$
\begin{gathered}
C^{6-i}= \\
\frac{\sigma_{\alpha}}{\sigma_{\alpha}+\left(\sigma_{b}-\sigma_{\alpha}\right)\left(\left(I_{2}^{6-i}\left(b_{1}\right)-I_{2}^{6-i}\left(\alpha_{1}\right)\right)\left(b_{1}^{2}+b_{2}^{2}+b_{3}^{2}-b_{i}^{2}\right)+\frac{\left(b_{1}^{2}+b_{2}^{2}+b_{3}^{2}-b_{i}^{2}\right) b_{1} b_{2} b_{3}}{\left(\alpha_{1}^{2}+\alpha_{2}^{2}+\alpha_{3}^{2}-\alpha_{i}^{2}\right) \alpha_{1} \alpha_{2} \alpha_{3}}\right)} \\
=\frac{\sigma_{\alpha}}{C_{2}^{6-i}}
\end{gathered}
$$

with $C_{2}^{6-i}$ defined in (2.21).

Obviously, the coincidence of the conductivities, which geometrically is reflected upon the absorption of the shell into the conductive core, i.e.,

$$
\sigma_{\alpha} \rightarrow \sigma_{b} \Leftrightarrow \alpha_{i} \rightarrow b_{i}, \quad i=1,2,3
$$

implies that

$$
\begin{gathered}
C^{6-i}=1, \quad i=1,2,3, \\
\tilde{\mathbf{N}}_{c}\left(b_{i}\right) \rightarrow \tilde{\mathbf{N}}_{c}\left(\alpha_{i}\right) \rightarrow \tilde{\mathbf{N}}\left(b_{i}\right)
\end{gathered}
$$

and finally,

$$
\mathbf{d}_{b} \rightarrow \mathbf{d}_{\alpha} \rightarrow \mathbf{d} .
$$

Then (3.58) is reduced to the corresponding expression for the magnetic induction field for the single homogeneous model 3 ]

$$
\mathbf{B}(\mathbf{r})=\frac{\mu_{0}}{4 \pi} \mathbf{d} \cdot\left(\frac{\mathbb{F}_{2}^{1}(\mathbf{r})}{\Lambda_{b}-\Lambda_{b}^{\prime}} \tilde{\mathbf{\Lambda}}-\frac{\mathbb{F}_{2}^{2}(\mathbf{r})}{\Lambda_{b}-\Lambda_{b}^{\prime}} \tilde{\mathbf{\Lambda}}^{\prime}-15 \sum_{\substack{i, j=1 \\ i \neq j}}^{3} x_{i} x_{j} I_{2}^{i+j}(\rho) \hat{\mathbf{x}}_{i} \otimes \hat{\mathbf{x}}_{j}\right)+O\left(e l_{3}\right) .
$$

4. Reduction to spherical geometry. It is hard, at a first glance, to accept that results on the ellipsoidal system do not reduce trivially to the corresponding results for the spherical system. Nevertheless, a more careful consideration helps to realize that this is an intrinsic difficulty which is due to the way the two systems are constructed. In particular, while the spherical system reduces down to a point, i.e. the center, the ellipsoidal system reduces to a two-dimensional manifold; that of the focal ellipse. As a consequence, the reduction from the ellipsoidal expressions to the spherical ones leads to complicated indeterminate forms which are not easy to handle. An appropriate, but not always obvious, grouping of terms is necessary to overcome these difficulties. This is a cumbersome process which will not be explained further here. As an example, we give the limit [3]

$$
\begin{aligned}
& \lim _{e \rightarrow s}\left(\frac{\mathbb{F}_{2}^{1}(\mathbf{r})}{\Lambda_{\alpha}-\Lambda_{\alpha}^{\prime}} \tilde{\mathbf{\Lambda}}-\frac{\mathbb{F}_{2}^{2}(\mathbf{r})}{\Lambda_{\alpha}-\Lambda_{\alpha}^{\prime}} \tilde{\mathbf{\Lambda}}^{\prime}-15 \sum_{\substack{i, j=1 \\
i \neq j}}^{3} x_{i} x_{j} I_{2}^{i+j}(\rho) \hat{\mathbf{x}}_{i} \otimes \hat{\mathbf{x}}_{j}\right) \\
& =\frac{\tilde{\mathbf{I}}}{r^{3}}-\frac{3}{r^{5}} \sum_{m=1}^{3} x_{m}^{2} \hat{\mathbf{x}}_{m} \otimes \hat{\mathbf{x}}_{m}-15\left(\frac{\mathbf{r} \otimes \mathbf{r}}{5 r^{5}}-\frac{1}{5 r^{5}} \sum_{m=1}^{3} x_{m}^{2} \hat{\mathbf{x}}_{m} \otimes \hat{\mathbf{x}}_{m}\right)=\frac{\tilde{\mathbf{I}}-3 \hat{\mathbf{r}} \otimes \hat{\mathbf{r}}}{r^{3}}
\end{aligned}
$$


where the notation $e \rightarrow s$ denotes the reduction of the ellipsoidal to the spherical system. More limits of this kind can be found in $\underline{3}$.

If we denote by $\alpha$ and $b$ the radii of the exterior and the interior reduced spheres respectively, then it is not hard to show that

$$
\begin{aligned}
& \lim _{e \rightarrow s} \Lambda_{\alpha}=\lim _{e \rightarrow s} \Lambda_{\alpha}^{\prime}=\alpha^{2}, \\
& \lim _{e \rightarrow s} h_{i}=0, \quad i=1,2,3, \\
& \lim _{e \rightarrow s} \mu=\lim _{e \rightarrow s} \nu=0, \\
& \lim _{e \rightarrow s} \rho=r,
\end{aligned}
$$

and finally that

$$
\lim _{e \rightarrow s} I_{2}^{m}(\rho)=\frac{1}{5 r^{5}}, \quad m=1,2,3,4,5 .
$$

Furthermore, the limits

$$
\begin{aligned}
& \lim _{e \rightarrow s} \tilde{\mathbf{M}}\left(b_{1}\right)=b^{2} \sum_{i=1}^{3} \hat{\mathbf{x}}_{i} \otimes \hat{\mathbf{x}}_{i}=b^{2} \tilde{\mathbf{I}}, \\
& \lim _{e \rightarrow s} \tilde{\mathbf{M}}\left(\alpha_{1}\right)=\alpha^{2} \tilde{\mathbf{I}} \\
& \lim _{e \rightarrow s} \tilde{\mathbf{N}}\left(b_{1}\right)=\frac{1}{2 b^{2}} \tilde{\mathbf{I}} \\
& \lim _{e \rightarrow s} C^{6-i}=\frac{1}{1+\left(\frac{\sigma_{b}}{\sigma_{\alpha}}-1\right)\left[\frac{3}{5}\left(\frac{b}{\alpha}\right)^{5}+\frac{2}{5}\right]}=C_{s}, \\
& \lim _{e \rightarrow s} \tilde{\mathbf{N}}_{c}\left(b_{1}\right)=\frac{C_{s}}{2 b^{2}} \tilde{\mathbf{I}} \\
& \lim _{e \rightarrow s} \tilde{\mathbf{N}}_{c}\left(\alpha_{1}\right)=\frac{C_{s}}{2 \alpha^{2}} \tilde{\mathbf{I}}
\end{aligned}
$$

imply that

$$
\begin{aligned}
& \lim _{e \rightarrow s} \mathbf{d}=\left(\mathbf{Q} \cdot \tilde{\mathbf{I}} \times \mathbf{r}_{0}\right) \cdot \frac{1}{2} \tilde{\mathbf{I}}=\frac{\mathbf{Q} \times \mathbf{r}_{0}}{2}, \\
& \lim _{e \rightarrow s} \mathbf{d}_{b}=\left(\mathbf{Q} \cdot \tilde{\mathbf{I}} \times \mathbf{r}_{0}\right) \cdot \frac{C_{s}}{2} \tilde{\mathbf{I}}=C_{s} \frac{\mathbf{Q} \times \mathbf{r}_{0}}{2}, \\
& \lim _{e \rightarrow s} \mathbf{d}_{\alpha}=\left(\mathbf{Q} \cdot \tilde{\mathbf{I}} \times \mathbf{r}_{0}\right) \cdot \frac{C_{s}}{2} \tilde{\mathbf{I}}=C_{s} \frac{\mathbf{Q} \times \mathbf{r}_{0}}{2},
\end{aligned}
$$

which they recover finally the known $[\underline{3}$. result for the sphere

$$
\lim _{e \rightarrow s} \mathbf{B}(\mathbf{r})=\frac{\mu_{0}}{8 \pi} \mathbf{Q} \times \mathbf{r}_{0} \cdot \frac{\tilde{\mathbf{I}}-3 \hat{\mathbf{r}} \otimes \hat{\mathbf{r}}}{r^{3}}+O\left(\frac{1}{r^{4}}\right) .
$$

Note that the spherical quadrupolic term on the right-hand side of (4.16) coincides with the corresponding result for a single homogeneous sphere and this is in accord with common knowledge that shells are not "readable" by MEG recordings. We remark here that this property of spherical geometry is due to the non-dependence of the radial 
component of the magnetic field on the electric potential [15. Our results though show that this is not true for the more realistic ellipsoidal model of the brain.

Appendix A. Ellipsoidal Harmonics. The reader can find the basic theory of ellipsoidal harmonics in the monumental work of Hobson [9]. Here, we provide only the expressions that will make the present work self-readable.

The interior Lamé functions $E_{n}^{m}(x)$ and the exterior Lamé functions $F_{n}^{m}(x)$, where $n=$ $1,2, \ldots$ denotes the degree and $m=1,2, \ldots, 2 n+1$ denotes the order of the corresponding function, solve the Lamé equation,

$$
\left(x^{2}-h_{2}^{2}\right)\left(x^{2}-h_{3}^{2}\right) E^{\prime \prime}(x)+x\left(2 x^{2}-h_{2}^{2}-h_{3}^{2}\right) E^{\prime}(x)+\left(A x^{2}+B\right) E(x)=0 .
$$

The constants $A$ and $B$ are appropriately associated with the degree $n$ and the order $m$. We only use the Lamé functions of degree less or equal to 2, and these are

$$
\begin{aligned}
& E_{0}^{1}(x)=1, \\
& E_{1}^{m}(x)=\left|x^{2}-\alpha_{1}^{2}+\alpha_{m}^{2}\right|^{\frac{1}{2}}, \quad m=1,2,3, \\
& E_{2}^{1}(x)=x^{2}-\alpha_{1}^{2}+\Lambda, \\
& E_{2}^{2}(x)=x^{2}-\alpha_{1}^{2}+\Lambda^{\prime} \\
& E_{2}^{6-m}(x)=\frac{E_{1}^{1}(x) E_{1}^{2}(x) E_{1}^{3}(x)}{E_{1}^{m}(x)}, \quad m=1,2,3,
\end{aligned}
$$

where the constants

$$
\left.\begin{array}{c}
\Lambda \\
\Lambda^{\prime}
\end{array}\right\}=\frac{1}{3} \sum_{n=1}^{3} \alpha_{n}^{2} \pm \frac{2}{3}\left[\sum_{n=1}^{3}\left(\alpha_{n}^{4}-\frac{\alpha_{1}^{2} \alpha_{2}^{2} \alpha_{3}^{2}}{\alpha_{n}^{2}}\right)\right]^{\frac{1}{2}}
$$

are the roots of

$$
\sum_{n=1}^{3} \frac{1}{\Lambda-\alpha_{n}^{2}}=0
$$

As Lamé showed, the harmonic eigensolutions in ellipsoidal form are given by the Lamé products

$$
\mathbb{E}_{n}^{m}(\rho, \mu, \nu)=E_{n}^{m}(\rho) E_{n}^{m}(\mu) E_{n}^{m}(\nu)
$$

which are regular at the origin, and the Lamé products

$$
\mathbb{F}_{n}^{m}(\rho, \mu, \nu)=F_{n}^{m}(\rho) E_{n}^{m}(\mu) E_{n}^{m}(\nu)
$$

which are regular at infinity.

The Lamé functions $E_{n}^{m}(\rho)$ and $F_{n}^{m}(\rho)$ are connected via the formula

$$
F_{n}^{m}(\rho)=(2 n+1) E_{n}^{m}(\rho) \mathrm{I}_{n}^{m}(\rho)
$$

where

$$
I_{n}^{m}(\rho)=\int_{\rho}^{+\infty} \frac{d t}{\left[E_{n}^{m}(t)\right]^{2} \sqrt{t^{2}-h_{2}^{2}} \sqrt{t^{2}-h_{3}^{2}}} .
$$


Note that the only difference of the factors $E_{n}^{m}$ in (A.9) is attributed to the interval of variation of the ellipsoidal coordinates $(\rho, \mu, \nu)$ which vary in the successive intervals

$$
-h_{3} \leq \nu \leq h_{3} \leq \mu \leq h_{2} \leq \rho<+\infty .
$$

The surface ellipsoidal harmonics $E_{n}^{m}(\mu) E_{n}^{m}(\nu)$ are orthogonal with respect to the weighting function

$$
l_{\rho}(\mu, \nu)=\frac{1}{\sqrt{\rho^{2}-\mu^{2}} \sqrt{\rho^{2}-\nu^{2}}}
$$

over the surface of any ellipsoid specified by a fixed $\rho>h_{2}$. The corresponding normalization constants, defined by (2.24) assume the values

$$
\begin{aligned}
& \gamma_{0}^{1}=4 \pi \\
& \gamma_{1}^{m}=\frac{4 \pi}{3} \frac{h_{1}^{2} h_{2}^{2} h_{3}^{2}}{h_{m}^{2}}, \quad m=1,2,3 \\
& \gamma_{2}^{1}=-\frac{8 \pi}{5}\left(\Lambda-\Lambda^{\prime}\right)\left(\Lambda-\alpha_{1}^{2}\right)\left(\Lambda-\alpha_{2}^{2}\right)\left(\Lambda-\alpha_{3}^{2}\right), \\
& \gamma_{2}^{2}=\frac{8 \pi}{5}\left(\Lambda-\Lambda^{\prime}\right)\left(\Lambda^{\prime}-\alpha_{1}^{2}\right)\left(\Lambda^{\prime}-\alpha_{2}^{2}\right)\left(\Lambda^{\prime}-\alpha_{3}^{2}\right),
\end{aligned}
$$

and

$$
\gamma_{2}^{6-m}=\frac{4 \pi}{15} h_{1}^{2} h_{2}^{2} h_{3}^{2} h_{m}^{2}, \quad m=1,2,3 .
$$

Usefull expressions for the gradients of the ellipsoidal harmonics, connection formulae between ellipsoidal harmonics and their Cartesian forms, as well as some basic relations among the elliptic integrals can be found in the appendices in [3].

Acknowledgments. The present work is part of a postdoctoral research project supported by the program "PYTHAGORAS" of the Greek Ministry of Education.

\section{REFERENCES}

[1] B.N. Cuffin and D. Cohen, "Magnetic Fields of a Dipole in Special Volume Conductor Shapes", IEEE Trans. Biomedical Eng., BME-24, pp. 372-381, 1997

[2] G. Dassios and F. Kariotou, "On the Geselowitz Formula in Biomagnetics", Quarterly of Applied Mathematics, 61 (2.2), 387-400, 2003 MR.1976377(2004d:78004)

[3] G. Dassios and F. Kariotou, "Magnetoencephalography in Ellispoidal Geometry", J. Math. Phys., 44, pp. 220-241, 2003 MR.1946700 (2004a:35204)

[4] G. Dassios and F. Kariotou, "On the Exterior Magnetic Field and Silent Sources in Magnetoencephalography", Abstract and Applied Analysis, 2004, no. 4, 307-314. MR2064143

[5] A.S. Fokas, Y. Kurylev and V. Marinakis, "The Unique Determination of Neuronal Currents in the Brain via Magnetoencephalography", Inverse Problems, 20, pp. 1067-1082, 2004 MR2087980 (2005c:92006)

[6] J.C. de Munck, "The Potential Distribution in a Layered Anisotropic Spheroidal Volume Conductor", J. Appl. Phys., 64, pp. 464-470, 1988

[7] D. B. Geselowitz, "On Bioelectric Potentials in an Inhomogeneous Volume Conductor", Biophys. J., 7, pp. 1-11, 1967

[8] D. B. Geselowitz, "On the Magnetic Field Generated Outside an Inhomogeneous Volume Conductor by Internal Current Sources", IEEE Trans. Magn., MAG-6, pp. 346-347, 1970

[9] E.W. Hobson, "The Theory of Spherical and Ellipsoidal Harmonics", Chelsea, New York, 1955 MR0064922 (16:356i) 
[10] R. J. Ilmoniemi, M. S. Hämäläinen and J. Knuutila, "The Forward and Inverse Problems in the Spherical model", pp. 278-282, in Biomagnetism: Applications and Theory, edited by Harold Weinberg, Gerhard Stroink, and Toivo Katila, Pergamon Press, New York, 1985

[11] Kamvyssas, G. and Kariotou, F., "Confocal Ellipsoidal Boundaries in EEG Modeling", Bulletin of the Greek Mathematical Society (in press)

[12] Kariotou, F., "Electroencephalography in Ellipsoidal Geometry", Journal of Mathematical Analysis and Applications, 290, pp. 324-342, 2004 MR2032245 (2004j:92033)

[13] J. Malmivuo and R. Plonsey, "Bioelectromagnetism", Oxford University Press, New York, 1995

[14] G. Nolte, T. Fieseler and G. Curio, "Perturbative Analytical Solutions of the Magnetic Forward Problem for Realistic Volume Conductors", J. Appl. Phys., 89, pp. 2360-2369, 2001

[15] J. Sarvas, "Basic Mathematical and Electromagnetic Concepts of the Biomagnetic Inverse Problem", Phys. Med. Biol., 32, pp. 11-22, 1987

[16] W.S. Snyder, M.R. Ford, G.G. Warner and H.L. Fisher, Jr., "Estimates of Absorbed Fractions for Monoenergetic Photon Sources Uniformly Distributed in Various Organs of a Heterogeneous Phantom", Journal of Nuclear Medicine, Supplement Number 3, August 1969, Volume 10, Pamphlet No. 5, Revised 1978 